\title{
A SURVEY OF RECENT IP LOOKUP SCHEMES $^{1}$
}

\author{
V. Srinivasan, G. Varghese \\ Microsoft Research, UCSD \\ cheenu@ccrc.wustl.edu,varghese@ccrc.wustl.edu
}

\section{Abstract}

Internet (IP) address lookup is a major bottleneck in high performance routers. IP address lookup is challenging because it requires a longest matching prefix lookup. It is compounded by increasing routing table sizes, increased traffic, higher speed links, and the migration to 128 bit IPv6 addresses.

We survey recent approaches to do fast IP lookups. We compare algorithms based on their lookup speed, scalability, memory requirement and update speed. While our main interest lies in the worst case lookup time, competitive update speeds and theoretical worst case bounds are also important. In particular we consider binary search on prefixes, binary search on prefix lengths, LC-tries, controlled prefix expansion and Lulea tries. We consider both software and hardware environments. We conclude that with these recent developments, IP lookups at gigabit speeds is a solved problem and that terabit lookup chips can be designed should the need arise.

\section{INTRODUCTION}

From the present intoxication with the Web to the future promise of electronic commerce, the Internet has captured the imagination of the world. It is hardly a surprise to find that the number of Internet hosts triple approximately every two years [7]. Also, Internet traffic is doubling every 3 months [28], partly because of increased users, but also because of new multimedia applications. The higher bandwidth need requires faster communication links and faster network routers. Gigabit fiber links are commonplace ${ }^{2}$, and yet the fundamental limits of optical transmission have hardly been approached. Thus the key to improved Internet performance is faster routers. This market opportunity has led 
to a flurry of startups (e.g., Avici, Juniper,Torrent) that are targeting the gigabit and terabit router market.

What are the fundamental impediments to improved router performance? An Internet message (called a packet) arrives at an input link. A processor $^{3}$ examines the destination address of the packet in a Forwarding Database to determine the output link. In some cases, the processor may also perform "firewall" transit checks that require a lookup in a database of firewall rules. If the checks succeed, the processor instructs a switching system to switch the packet the corresponding output link. Many routers also do a form of scheduling at the output link to ensure fairness among competing packet streams, and to provide delay bounds for time critical traffic such as video.

Thus the three central bottlenecks in router forwarding are lookups, switching, and output scheduling. Switching is well studied, and good solutions like fast busses and crossbar switches (e.g., [14, 30]) have been developed. Similarly, most vendors feel that full scale fair queuing ${ }^{4}$ is not required for a few years until video usage increases. In the interim, cheaper approximations such as weighted and deficit round robin [23] ensure fairness and can easily be implemented. So IP lookups remain a major bottleneck. In this survey, we look at recent algorithms for IP address lookups.

\section{THE INTERNET LOOKUP PROBLEM}

Internet address lookup would be simple if we could lookup a 32 bit IP destination address in a table that lists the output link for each assigned Internet address. In this case, lookup could be done by hashing, but a router would have to keep millions of entries. To reduce database size and routing update traffic, a router database consists of a smaller set of prefixes. This reduces database size, but at the cost of requiring a more complex lookup called longest matching prefix. It also requires a more complex update procedure when prefixes are added and deleted.

A metaphor can explain the compression achieved by prefixes. Consider a flight database in London. We could list the flights to a thousand U.S. cities in our database. However, suppose most flights to the U.S. hub though Boston, except flights to California that hub through LA. We can reduce the flight database from thousand entries to two prefix entries (USA $^{*} \rightarrow$ Boston; USA.CA.* $\rightarrow$ LA). We use "*" to denote a wildcard that can match any number of characters. The flip side of this reduc- 
tion is that a destination city like USA.CA.Fresno will now match both the USA* and USA.CA.* prefixes; we must return the longest match (USA.CA.*).

The Internet address lookup problem is similar except that that we use bit strings instead of character strings. The currently deployed version of the Internet ( $\mathrm{v} 4$ ) uses 32 bit destination addresses; each Internet router can have a potentially different set of prefixes, each of which we will denote by a bit string (e.g., $01^{*}$ ) of up to 32 bits followed by a "*". Thus if the destination address began with 01000 and we had only two prefix entries $\left(01^{*} \rightarrow \mathrm{L} 1 ; 0100^{*} \rightarrow \mathrm{L} 2\right)$, the packet should be switched to link L2.

The Internet began with a simpler form of hierarchy in which 32 bit addresses were divided into a network address and a host number, so that routers could only store entries for networks. For flexible address allocation, the network addresses came in variable sizes: Class A (8 bits), Class B (16 bits), and Class C (24 bits). Organizations that required more than 256 hosts were given class $\mathrm{B}$ addresses; these organizations further structured their Class B addresses ("subnetting") for internal routing. Finally, Class B addresses began to run out. Thus larger organizations needed multiple Class $\mathrm{C}$ addresses. To reduce backbone router table size, the CIDR scheme [21] now allocates larger organizations multiple contiguous Class $\mathrm{C}$ addresses that can be aggregated by a common prefix. In summary, the forces of subnetting and CIDR "supernetting" have led to the use of prefixes for the Internet. While the currently deployed IPv4 protocol uses 32 bit addresses, the next generation IPv6 protocol uses 128 bit addresses and continues to use longest matching prefix.

Best matching prefix is also used in the OSI Routing scheme [18]. Best matching prefix appears to be a fundamental problem for routers, and not just an artifact of the way the Internet has evolved.

\section{PERFORMANCE MODEL}

The choice of a lookup algorithm depends crucially on assumptions about the routing environment and the implementation environment. We also need a performance model with precise metrics to compare algorithms. 


\section{Routing Databases}

The Internet consists of local domains which are interconnected by a backbone consisting of multiple Internet Service Providers (ISPs). Accordingly, there are two interesting kinds of routers [1]: enterprise routers (used in a campus or organization) and backbone routers (used by ISPs). The performance needs of these two types of routers are different.

Backbone routers today [1] can have databases of up to 45,000 prefixes (growing every day, several of them with multiple paths). The prefixes contain almost all lengths from 8 to 32 ; however, because of the evolution from Class $\mathrm{B}$ and Class $\mathrm{C}$ addresses, there is considerable concentration at 24 and 16 bit prefix lengths. Because backbone routers typically run the Border Gateway Protocol [22], and some implementations exhibit considerable instability, route changes can occur up to 100 times a second $[1,11]$, requiring algorithms for handling route updates that take $10 \mathrm{msec}$ or less. Backbone routers may require frequent reprogramming as ISPs attempt to deal with customer requirements such as virus attacks. The distribution of packet sizes is bimodal, with peaks corresponding to either 64 byte control packets or 576 byte data packets.

Enterprise routers have smaller databases (up to 1000 prefixes) because of the heavy use of default routes for outside destinations. Routes are also typically much more stable, requiring route updates at most once every few seconds. The packet sizes are bimodal and are either 64 bytes or 1519 bytes $^{5}$. However, large multi-campus enterprise routers may look more like backbone routers.

Address space depletion has lead to the next generation of IP (IPv6) with 128 bit addresses. While there are plans for aggressive aggregation to reduce table entries, the requirement for both provider based and geographical addresses, the need for connections to multiple ISPs, plans to connect control devices on the Net, and the use of features like Anycast [3], all make it unlikely that backbone prefix tables will be smaller than in IPv4.

We use four publically available prefix databases for our comparisons. These are made available by the IPMA project [15] and are daily snapshots of the routing tables used at some major Network Access Points (NAPs). The largest of these, MaeEast (about 38,000 prefixes), is a reasonable model for a large backbone router; the smallest database, PAIX (around 713 prefixes), can be considered a model for an Enterprise router. We will compare lookup schemes using these four databases with respect to three metrics: search time (most crucial), storage, and update times. 


\section{Implementation Model}

In this paper, we will compare lookup algorithms using a software platform. Software platforms are more flexible and have smaller initial design costs. For example, BBN [6] uses DEC Alpha CPUs in each line card. However, hardware platforms are higher performance and cheaper after volume manufacturing. For example, Torrent [6] uses a hardware forwarding engine. We will briefly discuss hardware platforms in Section 6.

Thus for the majority of this paper, we will consider software platforms using modern processors such as the Pentium [9] and the Alpha [24]. These CPUs execute simple instructions very fast (few clock cycles) but take much longer (thirty to fifty clock cycles) to make a random access to main memory. The only exception is if the data is in either the Primary (L1) or Secondary Cache (L2), which allow access times of a few clock cycles. The distinction arises because main memory uses slow cheap Dynamic Memory (DRAM, 60-100 nsec access time) while cache memory is expensive but fast Static Memory (SRAM, 10-20 nsec). When a READ is done to memory of a single word, the entire cache line is fetched into the cache. This is important because the remaining words in the cache line can be accessed cheaply for the price of a single memory READ.

Thus an approximate measure of the speed of any lookup algorithm, is the number of main memory (DRAM) accesses required, because these accesses often dominate search times. To do so, we must have an estimate of the total storage required by the algorithm to understand how much of the data structures can be placed in cache. Finally, both cache accesses and clock cycles for instructions are important for a more refined comparison. To measure these, we must fix an implementation platform and have a performance tool capable of doing dynamic instruction counts that incorporate pipeline and superscalar effects.

We chose a commodity $300 \mathrm{Mhz}$ Pentium II running Windows NT that has a 8 Kbyte L1 data cache, a 512 KByte L2 cache, and a cache line size of 32 bytes. Since prefix databases are fairly large and the L1 cache is quite small, we (pessimistically) chose to ignore the effects of L1 caching. Thus our model assumes that every access leads to a L1 cache miss. When there is a L1 miss, the time to read in the first word in the cacheline from the L2 cache is 15 nsec. When there is a L2 miss, the total time to read in the word from memory (including the effects of the L2 miss) is 75 nsec. While many of our experiments use a 512 Kbyte L2 
cache size, we also present a few projected results assuming an L2 cache size of 1024 Kbytes.

We chose the Pentium platform because of the popularity of Wintel platforms, and the availability of useful tools. We believe the results would be similar if run on other comparable platforms such as the Alpha. We have implemented several previously known schemes on this platform. Further, we use a tool called Vtune [8] that gives us access to dynamic instruction counts, cache performance, and clock cycles for short program segments. Thus for careful analytical worst-case comparisons, we use speed measurements given to us by Vtune. In addition, we also do a test of average speed using accesses to a million randomly chosen IP addresses.

The analytic worst case measurements we use are much more conservative than what would be obtained by actually running the program on the Pentium II PC. This is because we assume that the L1 cache always misses, that branch prediction always fails, and that the worst possible branching sequence in the code is taken. A second reason for using Vtune is the difficulty of otherwise measuring the time taken for short code segments. A standard technique is to run $C$ lookups to the same address $D$, measure (using the coarse-grained system clock) the time taken for these lookups, and then to divide by $C$ to estimate the time taken for a single lookup. Unfortunately, such measurements are optimistic because repeated lookups to the same address are likely to result in the few relevant parts of the data structure (i.e., the parts used to lookup $D$ ) entering the L1 cache.

\section{EARLIER SCHEMES}

We describe previous schemes for IP lookup and compare them using our software performance model. We divide these schemes into four categories: conventional algorithms, hardware and caching solutions and protocol based solutions. For the rest of this paper, we use BMP as a shorthand for Best Matching Prefix and $W$ for the length of an address (32 for $\mathrm{v} 4$, and 128 for $\mathrm{v} 6$ ).

The most commonly available IP lookup implementation is found in the BSD kernel, and is a radix trie implementation [25]. If $W$ is the length of an address, the worst-case time in the basic implementation can be shown to be $O(W)$. Thus the implementation can require up to 32 or 128 worst-case costly memory accesses for IPv4 and IPv6 respectively. 
Even in the best case, with binary branching and 40,000 prefixes, this trie implementation can take $\log _{2}(40,000)=16$ memory accesses. A modified binary search technique is described in [12]. However, this method requires $O\left(\log _{2} 2 n\right)$ steps, with $n$ being the number of routing table entries. With 40,000 prefixes, the worst case would be 17 memory accesses. Using our crudest model, and 75 nsec DRAM, a trie or binary search scheme will take at least 1.2 usec.

Content-addressable memories(CAMs) that do exact matching can be used to implement best matching prefix. A scheme in [13] uses a separate CAM for each possible prefix length. This can require 32 CAMs for IPv4 and $128 \mathrm{CAMs}$ for IPv6, which is expensive. It is possible to obtain CAMs that allow "don't care" bits in CAM entries to be masked out. Such designs only require a single CAM. However, the largest such CAMs today only allow around 8000 prefixes. While such a CAM may be perfectly adequate for an enterprise router, it is inadequate for a backbone router. Finally, CAM designs have not historically kept pace with improvements in RAM memory. Thus any CAM solution runs the risk of being made obsolete in a few years by software running on faster processors and memory.

Caching has not worked well in the past in backbone routers because of the need to cache full addresses (it is not clear how to cache prefixes). This potentially dilutes the cache with hundreds of addresses that map to the same prefix. Also, typical backbone routers may expect to have hundreds of thousands of flows to different addresses. Some studies have shown cache hit ratios of around 50-70 percent [16]. Caching can help but does not avoid the need for fast lookups.

The main idea in Protocol Based solutions (IP and Tag Switching) is to replace best matching prefix by an exact match by having a previous hop router pass an index into the next router's forwarding table. This leads to a much faster lookup scheme (1 memory access), but the cost is additional protocol and potential set up delays. IP switching [16] relies on switching long lived flows. This solution may be ineffective with short-lived flows such as web sessions. Tag switching $[2,20]$ does not work at the boundaries of administrative domains. Both schemes require large parts of the network to make the required protocol changes before performance will improve. Both schemes also increase the vulnerability of an already fragile set of Internet routing protocols (see [11]) by adding a new protocol that interacts with every other routing protocol. Also, neither completely avoids the BMP problem. 


\section{NEW ALGORITHMS}

Several new techniques $[4,17,31,26]$ for best matching prefix were invented in the past two years. The expanded trie scheme [26] implements multibit tries based on what is called controlled prefix expansion. The Lulea Scheme [4] is based on implementing multibit tries but compresses trie nodes to reduce storage to fit in cache. While the worst case is still $O(W)$ memory accesses where $W$ is the address length, these accesses are to fast cache memory. The LC-trie scheme [17] is also based on implementing multibit tries but compresses them using what the authors call level compression.

The binary search on prefix lengths scheme [31] is based on binary search of the possible prefix lengths and thus takes a worst case of $\log _{2} W$ hashes, where each hash is assumed to be a single access to memory. It is much harder to determine the new schemes currently being designed or used by router vendors because they regard their schemes as trade secrets. However, Rapid City [6] and Torrent [29] use schemes based on hashing that claim good average performance but have poor worst case times (16 memory accesses for the Torrent ASIK scheme). A technique based on controlled prefix expansion for improving the worst case lookup performance of the binary search of prefix lengths was introduced in [26] and we refer to this scheme as 'Binary search on levels with expansion'.

Table 1 Prefix databases as on 12 Sep 97

\begin{tabular}{|l|r|r|}
\hline Database & $\begin{array}{r}\text { Number of } \\
\text { Prefixes }\end{array}$ & $\begin{array}{r}\text { Number of } \\
\text { 24 bit prefixes }\end{array}$ \\
\hline MaeEast & 38816 & 22872 \\
MaeWest & 14065 & 7850 \\
Pac & 3811 & 2455 \\
Paix & 713 & 377 \\
\hline
\end{tabular}

If we rule out pure caching and protocol based solutions, it is important to compare the other schemes using a common implementation platform and a common set of databases. We extracted the BSD lookup code using Patricia tries into our Pentium platform; we also implemented 6-way search [12] and binary search on hash tables [31] using the largest (MaeEast) database. We project the worst case evaluation presented in [4] and the average case numbers in [17] to the $300 \mathrm{Mhz}$ Pentium II platform with $15 \mathrm{nsec}$ L2 cache (recall that $15 \mathrm{nsec}$ is the time taken for the first word in the cacheline to be read when there is a L1 cache miss). Projections were done using the numbers reported in the papers 
describing the schemes, and by scaling the earlier results to account for the (faster) clock cycle times in our platform. The results are shown in Table 2. We also plot the worst case lookup times of various schemes in Figure 1.

Table 2 Lookup times for various schemes on a $300 \mathrm{MHz}$ pentium II for the MaeEast database. The times for binary search on hash tables are projected assuming good hash functions can be found. The average performance is determined by the time taken when the best matching prefix is a 24 bit prefix as there are very few prefixes with length 25 and above. Note that for the, Lulea scheme, the numbers have been recalculated with $300 \mathrm{MHz}$ clock and 15 nsec latency L2 cache. For binary search on levels, memory is estimated assuming minimal perfect hash functions and $\log _{2} W=5$ hashes. To be fair to the other schemes, we would like to point out that the numbers for our schemes are from highly optimized code and it might be possible to speedup other schemes simply by optimizing the implementation

\begin{tabular}{|l|r|r|r|}
\hline & $\begin{array}{r}\text { Average } \\
(24 \text { bit prefix } \\
\text { (nsec) }\end{array}$ & $\begin{array}{r}\text { Worst } \\
\text { case } \\
\text { (nsec) }\end{array}$ & $\begin{array}{r}\text { Memory required for } \\
\text { MaeEast database } \\
\text { (KBytes) }\end{array}$ \\
\hline Patricia trie & 1500 & 2500 & 3262 \\
LC trie scheme & 800 & 1000 & 700 \\
Binary Search & 250 & 650 & 1600 \\
on prefix lengths & & & \\
6-way search & 490 & 490 & 950 \\
on prefixes & & & \\
Lulea scheme & 349 & 409 & 160 \\
$\begin{array}{l}\text { Binary search } \\
\text { on prefix lengths } \\
\text { with expansion }\end{array}$ & 250 & 250 & 3200 \\
Expanded tries & & & \\
\hline
\end{tabular}

\section{Comparing Multibit Trie Schemes}

The Lulea scheme [4] uses a clever scheme to compress multibit trie nodes using a bitmap. It uses a trie with fixed strides of $16,8,8$; they are able to compress large trie nodes without slow linear search using a fast method of counting the number of bits set in a large bitmap. However, updating this bitmap is a slow operation. Also the Lulea approach pushes the prefix information to the leaves in the trie, which causes insertion to be $\mathrm{O}(\mathrm{N})$. The Lulea scheme also optimizes the information associated with prefixes by noting that there are (typically) only a small number of next hops. All these cause the Lulea scheme to have a slow insertion time. 


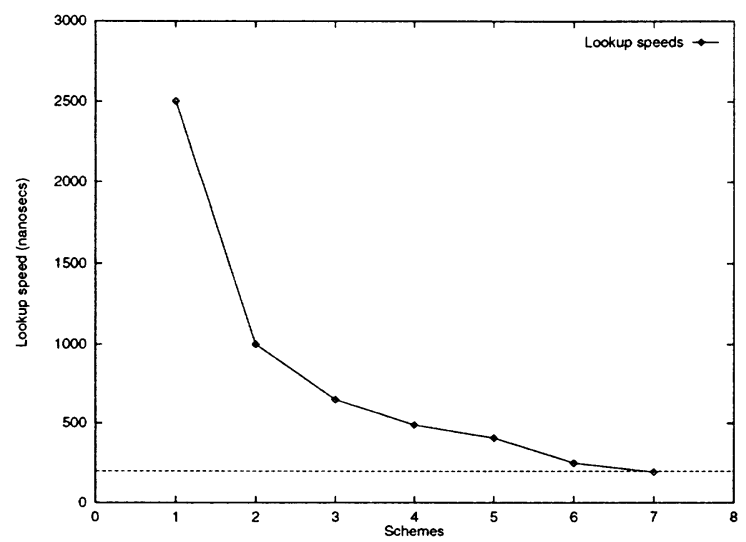

Figure 1 The lookup speeds of various schemes in nanoseconds. The scheme numbers used are 1. Patricia tries 2. LC-tries 3. Binary search on prefix lengths 4. Multiway search on prefixes 5 . Lulea tries 6 . Binary search prefix lengths with expansion and 7 . Expanded tries. The dotted line corresponds to a lookup speed of 200 nanoseconds.

When compared to these other schemes, expanded multibit trie schemes have greater tunability and faster insertion/deletion times. Both the Lulea [4] and binary search on prefix lengths [31] schemes are competitive in lookup speeds, but have an insertion cost of $\mathrm{O}(\mathrm{N})$. So we conclude that in a software environment, expanded tries give the best performance while allowing fast incremental updates.

\section{IP LOOKUPS IN HARDWARE}

While this paper has focused on software comparisons, it is also important to consider how IP lookup schemes could be implemented in hardware. Many vendors today are using hardware IP lookup engines because of the cheaper cost and the higher speeds offered by such engines. Thus in this section, we first present a hardware model and the relevant metrics for hardware. We then briefly consider the schemes described in this paper from a hardware implementation perspective.

We assume a cheap forwarding engine (say 50 dollars assuming high volumes) operating at a clock rate of 2-10 nsec. We assume the chip can place its data structure in SRAM (with say $10 \mathrm{nsec}$ access times) and/or DRAM (60-100 nsec access times). In memory comparisons, it is important to observe that SRAM is several times more expensive than DRAM. For example, following current prices, SRAM costs six times as much as DRAM per byte. Finally, some forwarding engines may have a 
few megabits of on-chip memory that is extremely fast (say 5 nsec access times).

Also, modern memory technologies like SDRAM and RAMBUS [19] provide a way to hide the long access times of DRAMs. They do so by providing a single chip with multiple DRAM banks to which accesses can be interleaved. Thus an access for bank $B 1$ can be started while the results of a previous access to bank $B 0$ are still forthcoming. Such technology lends itself naturally to pipelining, with the memory for each pipeline stage in a separate bank. At the same time, the forwarding engine only needs a single set of address pins to drive the multiple memory banks of a single RAMBUS or SDRAM.

Besides the search time measured in memory accesses, two other crucial measures for a hardware implementation are worst case memory usage and update times. While we have described the memory usage of different algorithms on "typical databases", it would be better to have a hardware implementation that can specify the worst case number of prefixes it can support, given a bounded amount of fast memory. For single chip forwarding engines, fast update times are also crucial. Besides the problem of backbone instability alluded to earlier, a chip that can do database updates entirely in hardware is much more attractive to customers. For example, consider an IP lookup chip that can do wire speed forwarding at OC-48 rates (one lookup every $166 \mathrm{nsec}$ ), can handle 50,000 arbitrary prefixes, and can do an update every msec. Such a chip would be extremely valuable today compared to CAM solutions that can only handle around 8000 prefixes. In Table 3 we present a comparison of the theoretical worst case bounds for lookup time, memory required and the update complexity of the schemes considered.

None of the schemes described in this paper have good worst case memory usage. However, it is easy to add worst case memory bounds for multibit tries using a technique called path compression, first described in [32]. In this technique, trie nodes with only one pointer are removed and substituted with an equivalent bit string. Path compression differs slightly from the more standard technique for compressing one-way branches using a skip count, is described in [10] and used in [25]. An important advantage of path compression over skip count compression is that path compression does not require backtracking [25, 27] during search.

Using path compression, the number of trie nodes used to store $n$ prefixes can be shown to be no more than $2 n$. Path compression [32] can be applied to all the multibit trie schemes considered in this survey to 
Table $3 \mathrm{~W}$ is the number of bits in the address and $\mathrm{N}$ is the number of prefixes in the database. For trie based schemes, $\mathrm{k}$ is the number of bits looked at in a single trie level. In the multiway search on prefixes, $\mathrm{k}$ is the degree of the search tree used.

\begin{tabular}{|l|r|r|r|}
\hline & $\begin{array}{r}\text { Worst case } \\
\text { lookup }\end{array}$ & Memory required & $\begin{array}{r}\text { Update } \\
\text { Complexity }\end{array}$ \\
\hline Multiway search & $O\left(\log _{k} N\right)$ & $O(N)$ & $O(N)$ \\
on prefixes & & & \\
Patricia trie & $O(W)$ & $O(N W)$ & $O(1)$ \\
LC-trie & $O(W / k)$ & $O\left(2^{k} N W / k\right)$ & $O\left(2^{k}\right)$ \\
Lulea trie & $O(W / k)$ & $O\left(2^{k} N W / k\right)$ & $O(N)$ \\
Expanded trie & $O(W / k)$ & $O\left(2^{k} N W / k\right)$ & $O\left(2^{k}\right)$ \\
Binary search & $O\left(\log _{2} W\right)$ & $O\left(N \log _{2} W\right)$ & $O(N)$ \\
on prefix lengths & & & \\
Binary search on & $O\left(\log _{2}(W / k)\right)$ & $O\left(N\left(\log _{2}(W / k)+2^{k}\right)\right)$ & $O\left(N 2^{k}\right)$ \\
prefix lengths & & & \\
with expansion & & & \\
\hline
\end{tabular}

provide worst case memory bounds.

A simple observation is that any search tree, whether a trie or a binary search tree, can be pipelined by splitting its levels into pipeline stages. The idea is that each level of the tree corresponds to a pipeline stage. The address is fed to the first stage which contains the root. The comparison at the first stage stage tells which pointer to follow (e.g., to node $N 1$ or $N 2$ ). The address is then passed to the second stage along with the pointer to say node $N 2$. The second stage contains both nodes $N 1$ and $N 2$. Since the passed pointer says $N 2$, the address is compared with $N 2$ and the resulting pointer is passed to the third stage, and so on. Pipelining allows a speed of one lookup per memory access at the cost of possibly increased complexity. As we have seen, memory technologies such as SDRAM and RAMBUS lend themselves naturally to pipelining, by providing a single set of pins that drive multiple memory banks. In summary, all the schemes described in this paper can be pipelined to provide one lookup per memory access.

Given this modification, the expanded trie schemes are best applicable to hardware implementations that use external DRAM. This is because DRAM is cheap and inexpensive, and the increased memory needs of expanded tries are not a significant issue. However, since even the fastest trie schemes take 3-4 memory accesses, implementations (e.g., routers that support OC-48 links) that require lookup times faster will require pipelining. As link speeds continue to increase (e.g., terabit forwarding), DRAM memory access times will become a bottleneck. At 
such speeds, the entire IP lookup database will need to be stored in SRAM or on-chip memory. In such environments, where memory is again limited, schemes that compress trie nodes such as [4] will do better than multibit expanded tries (with relevant modifications to support high update speeds).

\section{CONCLUSIONS}

We believe with [31] and [4] that IP lookup technology can be implemented in software, at least up to gigabit speeds. We also believe that fast lookup algorithms make the arguments for tag and IP switching less compelling. We also believe that terabit lookup chips can be designed if such a need should arise. Finally, we believe that routers of the future may be less vertically integrated than at present; instead they will be assembled from special chips for functions (e.g., lookups, switching, and scheduling) and commodity routing software, just as computers evolved from mainframes to PCs. We hope the lookup technology described by us and others will contribute to this vision of the future.

We would like to thank Sanjay Cherian (Ascend), Zubin Dittia (Washington University), Will Eatherton (Washington University), Chaitanya Kodeboyina (Microsoft), Geoff Ladwig (Bay Labs), Craig Partridge (BBN), and Marcel Waldvogel (ETH) for invaluable feedback.

\section{Notes}

${ }^{1}$ George Varghese is supported by NSF Grant NCR-9405444 and an ONR Young Investigator Award. This work was done while both authors were at Washington University, St. Louis. George Varghese is now a professor at the University of California, San Diego.

${ }^{2} \mathrm{MCI}$ and UUNET have upgraded their Internet backbone links to $622 \mathrm{Mbits} / \mathrm{sec}$

${ }^{3}$ Some designs have a processor per input link; others use a processor per router. Some designs use a general purpose processor; others use dedicated hardware.

${ }^{4}$ Fair queuing [5] is a form of output scheduling that guarantees bandwidth fairness and tight delay bounds.

${ }^{5} 576$ byte data packets arise in ISPs because of the use of a default size of 576 bytes for wide area traffic; 1519 byte size packets in the enterprise network probably arises from Ethernet maximum size packets

\section{REFERENCES}

[1] Scott Bradner. Next Generation routers Overview. Presented at Networld Interop'97, Atlanta, Georgia, 1997. 
[2] G. Chandranmenon and G. Varghese. Trading packet headers for packet processing. IEEE/ACM Transactions on Networking, (April):141-152, 1996.

[3] S. Deering and R. Hinden. Internet Protocol, Version 6 (IPv6) Specification RFC 1883. IETF http://www.ietf.org/rfc/, 1995.

[4] M. Degermark, A. Brodnik, S. Carlsson, and S. Pink. Small Forwarding Tables for Fast Routing Lookups. Proceedings SIGCOMM 97, (October):3-14, 1997.

[5] A. Demers, S. Keshav, and S. Shenker. Analysis and Simulation of a fair queuing algorithm. Proceedings of Sigcomm 89, (September):1-12, 1989.

[6] Craig Partridge et. al. A 50-Gb/s IP Router. IEEE/ACM Transactions on Networking, (June):237-248, 1998.

[7] M. Gray. Internet Growth Summary. http://www.mit.edu/people/ mkgray/net/ internet-growth-summary.html, 1996.

[8] Intel. VTune Performance Enhancement Environment. Intel Corporation, Santa Clara, CA, USA, 1997.

[9] Intel. Pentium Pro and Pentium II processors and related products. Intel Corporatation, Santa Clara, CA, USA, 1998.

[10] D. Knuth. Fundamental Algorithms vol 3: Sorting and Searching. AddisonWesley, 1973.

[11] C. Labovitz, G. Malan, and F. Jahanian. Internet Routing Instability. Proceedings of SIGCOMM 97, (October):115-126, 1997.

[12] B. Lampson, V. Srinivasan, and G. Varghese. IP Lookups using Multi-way and Multicolumn Search. Proceedings of IEEE Infocom 98, (April):1248-1256, 1998.

[13] A. McAuley, P. Tsuchiya, and D. Wilson. Fast multilevel heirarchical routing table using content-addressable memory. U.S. Patent serial number 034444, 1995.

[14] Nick McKeown, Martin Izzard, Adisak Mekkittikul, Bill Ellersick, and Mark Horowitz. The Tiny Tera: A Packet Switch Core. IEEE Micro, (January):26-33, 1997.

[15] Merit. Ipma statistics. http://nic.merit.edu/ipma, (Snapshot on 12 September 97), 1997.

[16] P. Newman, G. Minshall, and L. Huston. IP Switching and Gigabit Routers. IEEE Communications Magazine, (January):64-69, 1997.

[17] S. Nilsson and G. Karlsson. Fast Address Look-Up for Internet Routers. Presented at the IEEE Broadband Communications'98, Stuttgart, Germany, (April), 1998.

[18] R. Perlman. Interconnections, Bridges and Routers. Addison-Wesley, 1992.

[19] Rambus. http://www.rambus.com/, 1998. 
[20] Y. Rechter, B. Davie, D. Katz, E. Rosen, and G. Swallow. Cisco Systems' Tag Switching Architecture Overview. Technical Report RFC 2105, 1997.

[21] Y Rechter and T Li. An Architecture for IP Address Allocation with CIDR RFC 1518. IETF http://www.ietf.org/rfc/, 1993.

[22] Y. Rechter and T. Li. A Border Gateway Protocol 4 (BGP-4) RFC 1771. IETF http://www.ietf.org/rfc/, 1995.

[23] M. Shreedhar and G. Varghese. Efficient fair queuing using deficit round robin. IEEE/ACM Transactions on Networking, (June):375-385, 1996.

[24] Richard Sites and Richard Witek. Alpha AXP Architecture Reference Guide. Butterworth Heinemann, 1995.

[25] K. Sklower. A Tree-Based Routing Table for Berkeley Unix. Presented at the 1991 Winter Usenix Conference, Dallas TX, 1991.

[26] V. Srinivasan and G. Varghese. Faster Address Lookups using Controlled Prefix Expansion. ACM Transactions on Computer Systems, (February):1-40, 1999.

[27] W. Stevens. TCP/IP Illustrated, Volume 1 The Protocols. Addison-Wesley, 1994.

[28] A. Tammel. How to survive as an ISP. Presented at Networld Interop'97, Atlanta, Georgia, 1997.

[29] Torrent. Torrent Systems, Inc. Torrent Systems Cambridge MA, http://www.torrent.com/, 1998.

[30] J. Turner. Design of a Gigabit ATM Switch. Proceedings of IEEE Infocom 97, (March):2-11, 1997.

[31] M. Waldvogel, G. Varghese, J. Turner, and B. Plattner. Scalable High Speed IP Routing Lookups. Proceedings of SIGCOMM 97, (October):25-36, 1997.

[32] H. Wilkinson, G. Varghese, and N. Poole. Compressed prefix matching database searching. U.S. Patent 5781772, 1998. 